\title{
A monadicity theorem
}

\section{Francis Borceux and B.J. Day}

\begin{abstract}
A monadicity theorem is established for functors which satisfy the conditions of the "first isomorphism theorem" (following Linton's terminology). An application is made to the characterisation of certain types of algebraic categories generated by linear monads.
\end{abstract}

\section{Introduction}

In this article we establish that a functor $U: B \rightarrow C$ with a left adjoint is crudely monadic if it satisfies conditions analogous to those described by Linton [6] and there called the first isomorphism theorem. For certain types of algebraic category this monadicity theorem is an important alternative to the standard monadicity criteria of Beck [7].

In Section 2 we give a characterisation theorem based on the first isomorphism theorem for certain types of algebraic category generated by linear monads.

Throughout the article we suppose, unless otherwise stated, that $V=(V, \otimes, I, \ldots)$ is a complete and cocomplete symmetric monoidal closed category and that all categorical algebra is relative to this $U$. For terminology and notation we refer to Ei lenberg and Kelly [5] and Mac Lane $[7]$.

\section{The theorem}

DEFINITION 1.1. The first isomorphism theorem is said to hold for a functor $U: B \rightarrow C$ if

FIT 0: B has coequalisers and kernel pairs,

Received 24 May 1977. 
FIT 1: $f$ is a coequaliser in $B$ iff $U f$ is a coequaliser in $\mathcal{C}$,

FIT 2: $(f, g)$ is a kernel pair in $B$ if $(U f, U g)$ is a kernel pair in $\mathrm{C}$. //

THEOREM 1.2. If $C$ has coequalisers and kernel pairs and $F \rightarrow U: B \rightarrow C$, then $B$ is crudely monadic over $C$ if the first isomorphism theorem holds for $U$.

Proof. Let $T=(T, \mu, \eta)$ be the monad generated by the adjunction $(\varepsilon, \eta): F \dashv U: B \rightarrow \mathcal{C}$, and let $M: B \rightarrow \mathcal{C}^{\top}$ be the canonical comparison functor

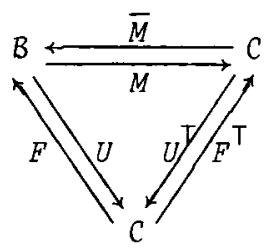

where $M B$ is $U B$ with structure $U \varepsilon_{B}: U F U B \rightarrow U B$. Because $B$ has coequalisers, $M$ has a left adjoint $\bar{M}$ defined by the following coequaliser:

$$
F U F C \stackrel{F \zeta}{\underset{\varepsilon_{F C}}{\longrightarrow}} F C \stackrel{e_{C}}{\longrightarrow} M(C, \zeta)
$$

and the counit $\bar{\varepsilon}$ is given by:

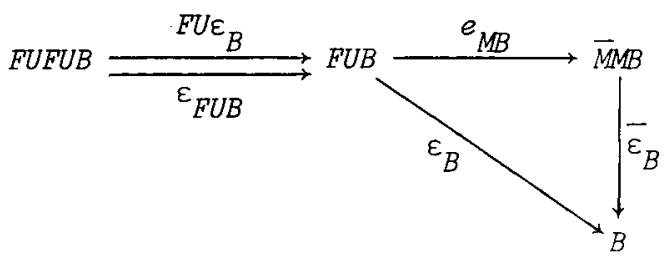

Applying $U$, we have that $U \varepsilon_{B}=\operatorname{coequ}\left(U F U \varepsilon_{B}, U \varepsilon_{F U B}\right)$. But $U e_{M B}$ is a coequaliser in $\mathcal{C}$ by hypothesis, so $U e_{M B}$ is an epimorphism, so $U \bar{\varepsilon}_{B}$ is an isomorphism. Thus $\bar{\varepsilon}_{B}$ is a coequaliser (by hypothesis) so $M$ reflects isomorphisms, so $U$ reflects isomorphisms, so $\bar{\varepsilon}_{B}$ is an isomorphism. Also $\bar{n}: 1 \rightarrow \vec{M}$ is given by 


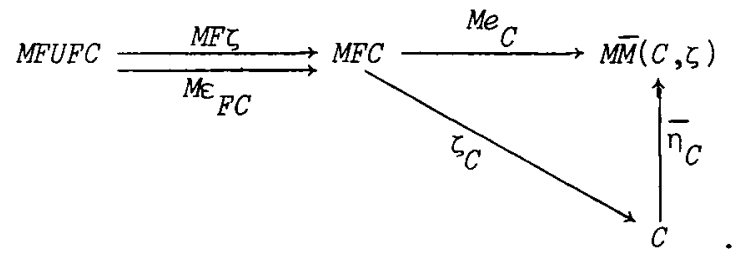

Let $\left(\phi_{1}, \phi_{2}\right)$ be the kernel pair of $e$ in $C$ (as in $C^{\top}$ ). The functor $U$ preserves kernel pairs, so $U e=\operatorname{coequ}\left(U \phi_{1}, U \phi_{2}\right)$ in $C$ by hypothesis. Thus $\bar{n}_{C}=\operatorname{coequ}\left(\zeta . U \phi_{1}, \zeta . U \phi_{2}\right)$ in $C$. Hence it remains to show that $\bar{n}_{C}$ is a monomorphism. Consider

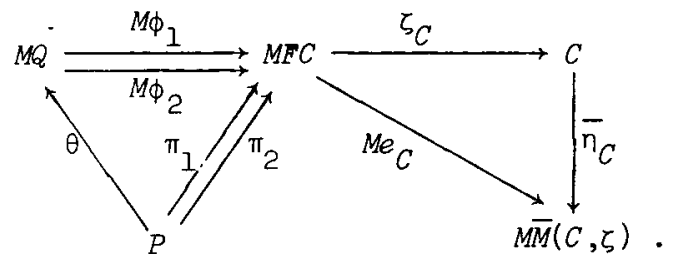

Let $\left(\pi_{1}, \pi_{2}\right)$ be the kernel pair of $\zeta_{C}$ in $C$ (as in $C^{\top}$ ). Then the comparison $\theta$ is a monomorphism and $M \phi_{1} \cdot \theta=\pi_{i}(i=1,2)$. But $\bar{M} \dashv M$, so we have

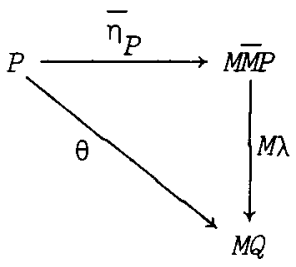

for a unique $\lambda: \bar{M} P \rightarrow Q$. Thus $\bar{\eta}_{P}$ is a monomorphism, hence is an isomorphism. Thus $\left(M \phi_{1} \cdot M \lambda, M \phi_{2} . M \lambda\right)$ is a kernel pair in $C^{\top}$, so, by hypothesis, $\left(\phi_{1} \cdot \lambda, \phi_{2} \cdot \lambda\right)$ is a kernel pair in $B$. Now, by definition of $\bar{\eta}, \lambda, \theta$, and $\alpha_{i}(i=1,2)$, the following diagram commutes $(i=1,2)$ : 


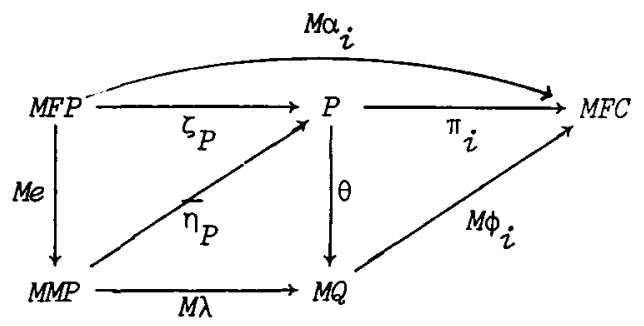

But $e=$ coequ $\left(\alpha_{1}, \alpha_{2}\right)$ in $B$ because $\bar{M}$ is a reflection, and coequ $\left(\alpha_{1}, \alpha_{2}\right)$ in $c^{\top}$ is just $(C, \zeta)$ because $\zeta_{P}$ is an epimorphism. Thus

$$
\begin{aligned}
e & =\operatorname{coequ}\left(\phi_{1} \lambda e, \phi_{2} \lambda e\right) \\
& =\operatorname{coequ}\left(\phi_{1} \lambda, \phi_{2} \lambda\right),
\end{aligned}
$$

since $e$ is an epimorphism. Hence $\lambda$ is an isomorphism because $\left(\phi_{1}, \phi_{2}\right)$ and $\left(\phi_{1} \lambda, \phi_{2} \lambda\right)$ are now both kernel pairs of $e$. Hence $\theta$ is an isomorphism, so $\bar{\eta}_{C}$ is an isomorphism. //

COROLLARY 1.3. If $\mathrm{C}$ has coequatisers and kernel pairs and $F \rightarrow U: B \rightarrow C$ is an adjunction such that $U F$ preserves coequalisers of reflective pairs then $B$ is crudely monadic over $C$ if and only if the first isomorphism theorem holds on $U$. I/

\section{Linear monads}

Let $N: A \rightarrow C$ be a fully faithful dense functor. Following Diers [4], we say that an $N$-theory $(T, t)$ is algebraic if $t^{o p}: A \rightarrow T^{o p}$ has a right $N$-adjoint $R$. We call an algebraic theory strictly $N$-hyperlinear if the mean tensor product (see [1]) $C(N A, C) * R$ tA exists in $\mathcal{C}$ and is $N$-absolute.

As usual, the category $c^{b}$ of $T$-algebras is defined by the pullback:

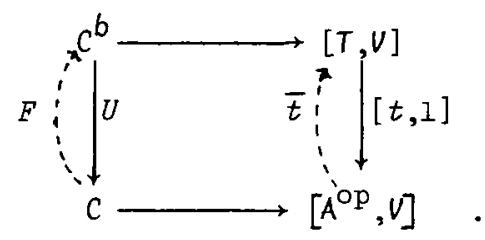


As a consequence

$$
\begin{aligned}
& {[t, 1] \bar{t}(\mathcal{C}(N-, C))=\int^{A} T(t A, t-) \otimes \mathcal{C}(N A, C)} \\
& \quad \cong \int^{A} \mathcal{C}(N-, R t A) \otimes C(N A, C) \cong \mathcal{C}(N-, \mathcal{C}(N A, C) * R t A) .
\end{aligned}
$$

So we obtain $F \dashv U$ where $U F$ is just the restriction of $\bar{t} \rightarrow[t, 1]$ to C.

$$
U F C=C(N A, C) * R t A
$$

Thus RtA $\cong$ UFNA and

$$
\mathcal{C}(N B, U F C) \cong \int^{A} \mathrm{C}(N A, C) \otimes C(N B, R t A) \cong \int^{A} \mathrm{C}(N A, C) \otimes \mathrm{C}(N B, U F N A)
$$

Thus the monad $T=U F$ is strictly $N$-hyperlinear in the sense of Day [3]. Such an algebraic category $c^{b}$ will be called strongly $N$-algebraic.

THEOREM 2.1. If $C$ has coequalisers and kermel pairs and each $C(N A,-), A \in A$, preserves coequalisers of reflective pairs, then a category $B$ is strongly $N$-algebraic over $C$ if and only if there exists $F \rightarrow U: B \rightarrow C$ such that

(1) $\int^{A} \mathrm{C}(N A, C) \otimes C(N B, U F N A) \cong \mathrm{C}(N B, U F C)$,

(2) the first isomorphism theorem holds for $U$.

Proof. Necessity follows from the fact that $\mathcal{C}(N A,-), A \in A$, preserves coequalisers of reflective pairs, thus $U F \cong C(N A,-) * U F A$ preserves coequalisers of reflective pairs, thus $U$ creates coequalisers of reflective pairs. For sufficiency we have that $U$ is monadic by (2) and Theorem 1.2. By density of $N$ we have $\int^{A} \mathcal{C}(N A, C) \cdot U F N A \cong U F C$ from (1). Thus the monad generated by the theory which maps $A^{\text {op }}$ to the full image of FN (see Day [2]) coincides with the monad $U F$, as required. //

This result should be compared with Lawvere's characterisation theorem (Linton [6], Corollary to Proposition 6) for the case $c=$ Ens. 


\section{References}

[1] Francis Borceux and G.M. Kelly, "A notion of limit for enriched categories", Buzl. Austral. Math. Soc. 12 (1975), 49-72.

[2] B.J. Day, "Linear monads", Bulz. Austral. Math. Soc. 17 (1977), $177-192$.

[3] B.J. Day, "On the rank of free monads" (Preprint, Department of Pure Mathematics, University of Sydney, Sydney, 1977).

[4] Y. Diers, "Foncteur pleinement fidèle dense classant les algèbres", (Publications Internes de I'U.E.R. de Mathématiques Pures et Appliquées, 58. Université des Seiences et Techniques de Lille I, 1975).

[5] Samuel Ei lenberg and G. Max Kelly, "Closed categories", Proc. Conf. Categorical Algebra, La Jolla, California, 1965, 421-562 (Springer-Verlag, Berlin, Heidelberg, New York, 1966).

[6] F.E.J. Linton, "Some aspects of equational categories", Proc. Conf. Categorical Algebra, La Jolla, California, 1965, 84-94 (SpringerVerlag, Berlin, Heidelberg, New York, 1966).

[7] S. Mac Lane, Categories for the working mathematician (Graduate Texts in Mathematics, 5. Springer-Verlag, Berlin, Heidelberg, New York, 1971).

Inistitut de Mathématique pure et appliquée, Université Catholique de Louvain,

Belgium;

Department of Pure Mathematics, University of Sydney, Sydney, New South Wales. 